\title{
Study of bacterial and fungal community structures in traditional koumiss from Inner Mongolia
}

\author{
Liang Guo, ${ }^{*}$ Mei Ya, Yuan-Sheng Guo, Wei-Liang Xu, Chun-Dong Li, Jian-Ping Sun, Jian-Jun Zhu, \\ and Jun-Ping Qian \\ Xilingol Vocational College, Xilin Gol Institute of Bioengineering, Xilin Gol Food Testing and Risk Assessment Center, Xilinhot 026000 , \\ Inner Mongolia, China
}

\section{ABSTRACT}

Koumiss is notable for its nutritional functions, and microorganisms in koumiss determine its versatility. In this study, the bacterial and fungal community structures in traditional koumiss from Inner Mongolia, China, were investigated. Our results demonstrated that 6 bacterial phyla represented by 126 genera and 49 species and 3 fungal phyla represented by 59 genera and 57 species were detected in 11 samples of artisanal koumiss. Among them, Lactobacillus was the predominant genus of bacterium, and Kluyveromyces and Saccharomyces dominated at the fungal genus level. In addition, there were no differences in the bacterial and fungal richness and diversity of koumiss from 3 neighboring administrative divisions in Inner Mongolia, and the bacterial and fungal community structures (the varieties and relative abundance of bacterial and fungal genera and species) were clearly distinct in individual samples. This study provides a comprehensive understanding of the bacterial and fungal population profiles and the predominant genus and species, which would be beneficial for screening, isolation, and culture of potential probiotics to simulate traditional fermentation of koumiss for industrial and standardized production in the future.

Key words: koumiss, bacterial, fungal, diversity

\section{INTRODUCTION}

Dairy products have been acknowledged as highly nutritious foods around the world. Koumiss, which originates from traditional fermentation of mare milk, is a very popular dairy foodstuff for the people of Mongolia, Kazakhstan, Kirgizstan, and some regions of Russia and Bulgaria (Danova et al., 2005). In China, koumiss has been used by the nomads who inhabit In-

Received June 4, 2018.

Accepted November 9, 2018.

*Corresponding author: herdman86@163.com ner Mongolia as a traditional Mongolian medicine to treat and heal intestinal dyspepsia, hypertension, and dyslipidemia (Rong et al., 2015; Gesudu et al., 2016).

Koumiss is produced by spontaneously fermenting mare milk in various containers made of wood, animal skin, and porcelain. The natural, homemade, palm-sized cloth bag that contains microbial flora from the previous year's koumiss serves as microbial starter culture for fermenting during horse lactation (June to September) during the current year. Fermentation continues spontaneously at ambient temperature $\left(\sim 20^{\circ} \mathrm{C}\right)$ for 1 to $3 \mathrm{~d}$, and fermented mare milk is beaten and stirred with a wooden stick to remove carbon dioxide, ensure homogeneity, and speed up spontaneous fermentation for eliminating propagation of pathogens. According to personal preference (organoleptic evaluation of acidity and alcohol content) and commercial needs, koumiss is bottled and stored at $4^{\circ} \mathrm{C}$ to extend its shelf life. In view of the artisanal technique of production of koumiss, spontaneous fermentation, which is propelled by natural microbial flora, determines its nutritional and functional profiles. Nevertheless, the microbial investigation of koumiss has been the subject of a limited number of studies. Lactobacillus casei Zhang (Ya et al., 2008), Lactobacillus fermentum SM-7 (Pan et al., 2011), Lactococcus lactis KLDS4. 0325 (Yang et al., 2014), Lactobacillus helveticus NS8 (Rong et al., 2015), and Lactobacillus crustorum MN047 (Yi et al., 2016), which were isolated from koumiss, demonstrated versatile probiotic attributes such as immunomodulation, cholesterol-lowering effects, inhibition of angiotensinconverting enzyme, anti-inflammatory function, and antibacterial activity. Moreover, the bacterial flora and yeasts in koumiss were isolated and identified using culture-independent methods, such as denaturing gradient gel electrophoresis, and species-specific PCR (Hao et al., 2010; Mu et al., 2012). Nonetheless, the bacterial and fungal community structures need to be simultaneously investigated in traditional koumiss. The comprehensive understanding of bacterial and fungal population profiles will be beneficial for simulating 
spontaneous fermentation of koumiss in laboratories and pilot plants for commercial production of artisanal koumiss.

The objective of this study was to investigate the bacterial and fungal community structures and to discover the predominant microbial genus and species during spontaneous fermentation of koumiss in Inner Mongolia. The conducted research would provide fundamental references for screening, isolation, and culture of microbial candidates with probiotic functions to simulate traditional fermentation of koumiss for industrial and standardized production in the future.

\section{MATERIALS AND METHODS}

\section{Collection of Samples}

Traditional production of koumiss originated from spontaneous fermentation of mare milk in Inner Mongolia. A total of 11 samples of artisanal koumiss were obtained from yurts of the Mongolian nomads across the entire grassland of Inner Mongolia, and 3 samples of mare milk (X0, H0, and $\mathrm{C} 0$ ) were obtained from 3 administrative divisions of Inner Mongolia during horse lactation (June to September). Koumiss samples were also derived from these 3 areas: 4 samples $(\mathbf{X} 1, \mathbf{X} 2$, X3, and X4) from Xilin Gol, 4 samples (H1, H2, H3, and H4) from Hulun Buir, and 3 samples (C1, C2, and C3) from Chifeng (Figure 1). The precise locations of sampling were Xilinhot, West Sunit Banner, West Ujimuqin Banner, and Abag Banner in Xilin Gol for $\mathrm{X} 1$ to X4, respectively; Hailar, Evenki Banner, New Barag Right Banner (West Banner), and New Barag Left Banner (East Banner) in Hulun Buir for H1 to H4, respectively; and Tianshan Town, Daban Town, and Hexigten Banner in Chifeng for C1 to C3, respectively. In addition, these sampling spots covered most areas of prairie in Inner Mongolia. These raw and fermented dairy products $(1,000 \mathrm{~mL})$ were collected into sterile, self-sealing bags as soon as possible, and bags were immediately placed on ice, stored at $4^{\circ} \mathrm{C}$, and analyzed to investigate nutritional components. After this collec-

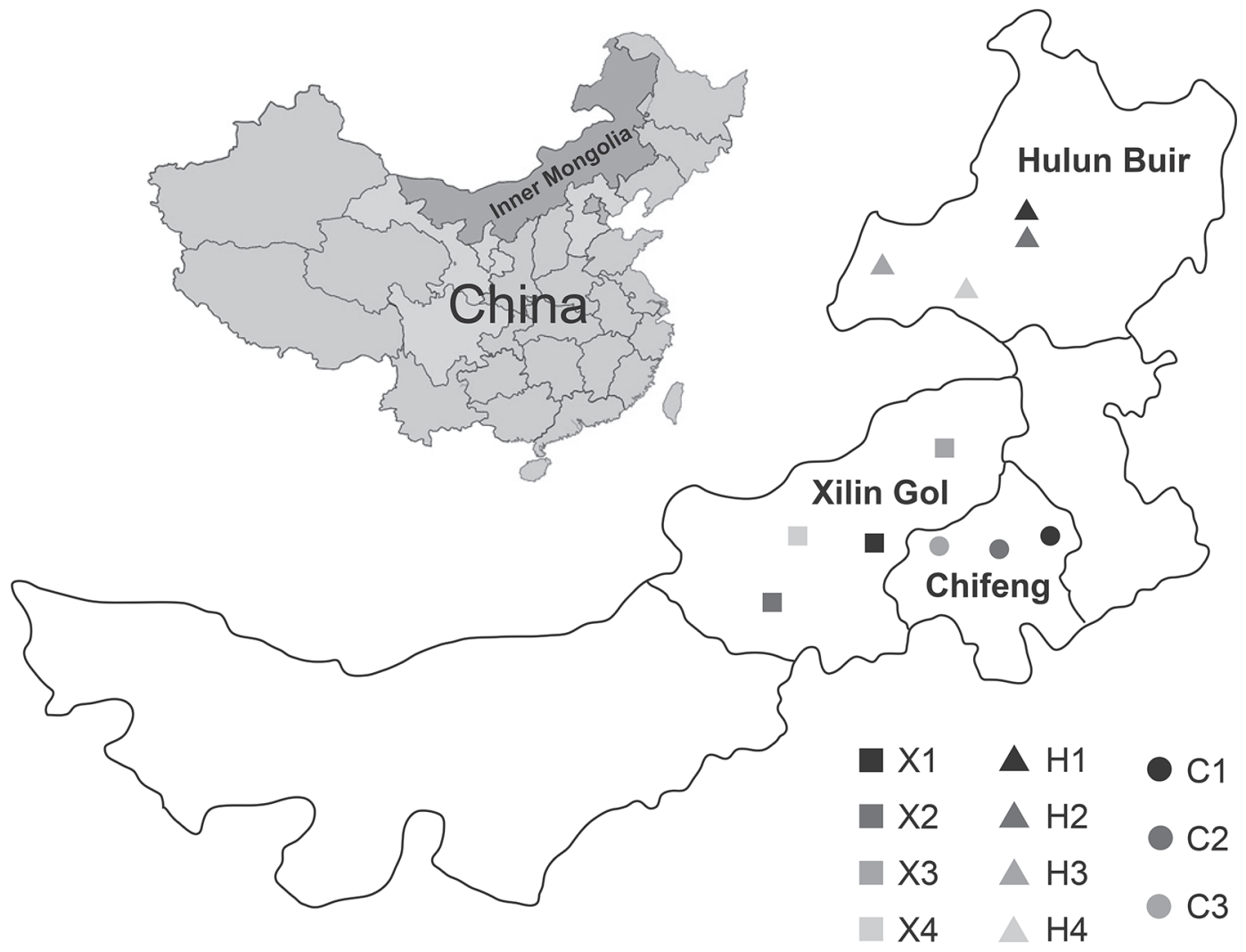

Figure 1. Sampling of koumiss from 3 administrative divisions of Inner Mongolia. The geographical position of Inner Mongolia in China is highlighted in black on the top left figure. The precise locations of sampling of X1, X2, X3, and X4 were Xilinhot, West Sunit Banner, West Ujimuqin Banner, and Abag Banner, respectively, in Xilin Gol. The locations of sampling of H1, H2, H3, and H4 were Hailar, Evenki Banner, New Barag Right Banner (West Banner), and New Barag Left Banner (East Banner), respectively, in Hulun Buir. The locations of sampling of C1, C2, and C3 were Tianshan Town, Daban Town, and Hexigten Banner, respectively, in Chifeng. 
tion of 11 samples from 3 different areas, the koumiss was subpackaged and stored at $-80^{\circ} \mathrm{C}$ for analysis of microbial community structure.

\section{Measurement of Lactose, Acidity, pH, Alcohol, Protein, and Fat of Mare Milk and Koumiss}

The content of lactose (China National Food Safety Standard, 2010), acidity (China National Food Safety Standard, 2016a), and alcohol (China National Food Safety Standard, 2016b) was measured according to Chinese national food safety standards. The content of lactose was calculated using the titration method with Feline's solution (Sinopharm, Beijing, China) and methylene blue (Sinopharm). The acidity of dairy product was determined by titration method with phenolphthalein indicator. The $\mathrm{pH}$ value of mare milk and koumiss was quantified by a $\mathrm{pH}$ meter. The content of protein was determined using the Kjeldahl method. The content of fat was measured using the Soxhlet extraction method.

\section{Microbial DNA Extraction and PCR Amplification}

Microbial DNA was extracted from koumiss using an EZNA stool DNA kit (Omega Bio-tek, Norcross, GA) according to the manufacturer's protocols. The V3-V4 region of the $16 \mathrm{~S}$ rRNA gene was amplified by PCR using primers 341F 5'-CCTACGGGNGGCWGCAG-3' and 806R 5'-GGACTACHVGGGTATCTAAT-3', where the barcode was an 8-base sequence to each sample. The internal transcribed spacer (ITS) region of the rRNA gene was amplified by PCR using primers ITS3-KYO2F 5'-GATGAAGAACGYAGYRAA-3' and ITS4R 5'-TCCTCCGCTTATTGATATGC-3', where the barcode was an 8-base sequence to each sample. The PCR reaction was performed in a mixture containing $5 \mu \mathrm{L}$ of $10 \times$ KOD buffer (Toyobo, Osaka, Japan), 5 $\mu \mathrm{L}$ of $2.5 \mathrm{~m} M \mathrm{dNTPs}$ (Toyobo), $1.5 \mu \mathrm{L}$ of each primer $(5 \mu M), 1 \mu \mathrm{L}$ of KOD polymerase (Toyobo), and 100 ng of template DNA extracted from koumiss and was executed under the following program: $95^{\circ} \mathrm{C}$ for $2 \mathrm{~min}$; 27 cycles of $98^{\circ} \mathrm{C}$ for $10 \mathrm{~s}, 62^{\circ} \mathrm{C}$ for $30 \mathrm{~s}$, and $68^{\circ} \mathrm{C}$ for 30 $\mathrm{s}$; and a final extension at $68^{\circ} \mathrm{C}$ for $10 \mathrm{~min}$.

\section{Illumina Hiseq2500 Sequencing}

Amplicons were extracted from 2\% agarose gel and purified using AxyPrep DNA Gel Extraction Kit (Axygen Biosciences, Union City, CA) according to the manufacturer's instructions and quantified using QuantiFluor-ST (Promega, Madison, WI). Purified amplicons were pooled in equimolar quantities and paired-end sequenced $(2 \times 250)$ by Illumina (San Diego, CA) platform according to standard protocols.

\section{Bioinformatics and Statistical Analysis}

Raw data containing adapters or low-quality reads would affect the assembly and analysis. Thus, to get high-quality clean reads, raw reads were further filtered after removing the reads containing more than $10 \%$ unknown nucleotides and less than $80 \%$ bases with quality value $>20$. Paired-end clean reads were merged as raw tags using FLASH version 1.2.11 (Magoč and Salzberg, 2011) with a minimum overlap of $10 \mathrm{bp}$ and mismatch error rates of $2 \%$. Noisy sequences of raw tags were filtered by QIIME version 1.9.1 (Caporaso et al., 2010) pipeline under specific filtering conditions (Bokulich et al., 2013) to obtain high-quality clean tags. Clean tags were searched against the reference database (http://drive5.com/uchime/uchim_download .html) to perform reference-based chimera checking using the UCHIME algorithm (http://www.drive5.com/ usearch/manual/uchime_algo.html). All chimeric tags were removed, and some effective tags were finally obtained for further analysis. The effective tags were clustered into the operational taxonomic units (OTU) of $\geq 97 \%$ similarity using the UPARSE pipeline (Edgar, 2013). The tag with the highest abundance was selected as a representative sequence within each cluster. Venn analysis was performed in $\mathrm{R}$ (https://cran.r-project .org/doc/manuals/) to identify unique and common OTU. The representative sequences were classified into organisms by the naive Bayesian model using RDP Classifier version 2.2 (Wang et al., 2007) based on the SILVA (Pruesse et al., 2007) database (https://www .arb-silva.de/) for $16 \mathrm{~S}$ rRNA sequencing and based on the UNITE (Köljalg et al., 2005) database (https:// unite.ut.ee/) for ITS sequencing. The OTU rarefaction curve, Chao1, Simpson, and all other alpha diversity indices were calculated in QIIME. Weighted and unweighted UniFrac distance matrixes were generated by QIIME. The principal coordinates analyses (PCoA) of weighted and unweighted UniFrac distances were calculated and plotted in R. Alpha and beta indices among groups were computed by the Kruskal-Wallis $\mathrm{H}$ test and Tukey's honest significant difference (HSD) test in $\mathrm{R}$.

\section{RESULTS AND DISCUSSION}

\section{Nutritional Characteristics of Traditional Koumiss from Inner Mongolia}

The content of lactose in mare milk was $7.58 \pm 0.34 \%$ and in koumiss was $3.51 \pm 2.54 \%$ for Xilin Gol, $3.66 \pm$ 
$2.32 \%$ for Hulun Buir, and $3.18 \pm 1.06 \%$ for Chifeng (Table 1). The level of lactose significantly decreased during spontaneous fermentation of mare milk $(P<$ $0.01)$. The characteristic of the high level of lactose in mare milk suggests that it is a valuable substrate for utilization by lactic acid bacteria and yeasts in the production of fermented dairy products (e.g., koumiss). Koumiss is a traditional drink usually made from mare milk by spontaneous fermentation of lactose to lactic acid and alcohol by lactic acid bacteria and yeasts. The acidity of mare milk was $4.39 \pm 0.23 \%$, and the acidity of koumiss was $119 \pm 46 \%$ for Xilin Gol, $226 \pm 114 \%$ for Hulun Buir, and $184 \pm 89 \%$ for Chifeng (Table 1). The content of alcohol in mare milk was $0 \%$ and in koumiss was $1.03 \pm 1.04 \%$ for Xilin Gol, $0.46 \pm 0.31 \%$ for Hulun Buir, and $1.07 \pm 1.05 \%$ for Chifeng (Table 1). The acidity and the alcoholic content significantly increased during spontaneous fermentation of mare milk $(P<$ 0.01 ). In addition, as shown in Table 1 , the contents of protein and fat were uniform during production of koumiss from mare milk $(P>0.05)$. The dynamics of nutritional components during spontaneous fermentation of mare milk implied that these samples were appropriate to be authentic koumiss, with more acid and alcohol as well as less lactose compared with raw mare milk, for the following analyses of bacterial and fungal community structures.

\section{Bacterial and Fungal Sequence Abundance and Diversity}

A total of 314,728 bacterial and 136,687 fungal highquality sequences were generated from 11 koumiss samples, with an average of 28,612 $(\mathrm{SD}=4,591)$ and $12,426$ ( $\mathrm{SD}=2,980)$ sequences for each sample, re- spectively. The average lengths of bacterial and fungal sequences were 462 and $373 \mathrm{bp}$, respectively. The highquality bacterial and fungal sequences were clustered into the OTU of $\geq 97 \%$ similarity. As shown in Table 2, at a genetic distance of $3 \%$, a total of 2,725 bacterial and 919 fungal OTU were identified from 11 koumiss samples, and the number of OTU per sample ranged from 178 to 340 for bacteria and 50 to 102 for fungi. Taxonomic classification was inferred from the closest matches of OTU to particular phylogenetic groups. Of the bacterial sequences, 93.4, 84, and $16.2 \%$ were assigned to phylum, genus, and species, respectively, for bacterial taxonomy. Of the fungal sequences, 96.9, 77.8, and $55.4 \%$ were assigned to phylum, genus, and species, respectively, for fungal taxonomy. More and more unclassified genus and species that were identified in the bacterial and fungal sequences resulted in decreasing in sequence assignment from phylum to species. From the analysis of alpha diversity for the bacterial and fungal sequences from 11 koumiss samples, the Shannon diversity curves, but not the rarefaction curves, reached the saturation phase (Figure 2). These results showed that the majority of bacterial and fungal phylotypes for samples had already been captured in the analysis, although some additional new bacterial and fungal phylotypes could possibly be identified by increasing the depth of sequencing. In addition, Good's coverage (values $>99 \%$ ) suggested that the majority of microbial phylotypes obtained from koumiss had already been captured.

In this study, the purpose of the Chao1 analysis was to evaluate the bacterial and fungal richness of koumiss, and the Shannon and Simpson indices were applied to indicate the bacterial and fungal diversities of koumiss (Table 2). The bacterial and fungal richness of koumiss

Table 1. Nutritional profiles of mare milk and traditional koumiss from Inner Mongolia

\begin{tabular}{|c|c|c|c|c|c|c|c|c|}
\hline Type & Location & Sample & $\begin{array}{l}\text { Lactose } \\
(\mathrm{g} / 100 \mathrm{~g})\end{array}$ & $\begin{array}{c}\text { Acidity }{ }^{1} \\
\left({ }^{\circ} \mathrm{T}\right)\end{array}$ & $\mathrm{pH}$ & $\begin{array}{c}\text { Alcohol }^{2} \\
(\%, \mathrm{vol} / \mathrm{vol})\end{array}$ & $\begin{array}{l}\text { Protein } \\
(\mathrm{g} / 100 \mathrm{~g})\end{array}$ & $\begin{array}{c}\text { Fat } \\
(\mathrm{g} / 100 \mathrm{~g})\end{array}$ \\
\hline \multirow[t]{2}{*}{ Mare milk } & Xilin Gol & X0 & 7.14 & 4.06 & 7.05 & 0 & 1.65 & 0.66 \\
\hline & Hulun Buir & Ho & 7.96 & 4.57 & 6.89 & 0 & 1.92 & 0.39 \\
\hline \multirow[t]{9}{*}{ Koumiss } & Xilin Gol & $\mathrm{X} 1$ & 0.32 & 188 & 3.39 & 2.4 & 2.03 & 1.50 \\
\hline & & $\mathrm{X} 2$ & 6.54 & 95 & 3.89 & 0 & 1.83 & 1.09 \\
\hline & Hulun Buir & H1 & 6.47 & 97 & 4.02 & 0 & 2.56 & 0.21 \\
\hline & & $\mathrm{H} 2$ & 4.23 & 165 & 3.30 & 0.8 & 1.88 & 0.61 \\
\hline & & H3 & 2.99 & 301 & 3.21 & 0.5 & 1.92 & 0.74 \\
\hline & & $\mathrm{H} 4$ & 0.94 & 340 & 3.30 & 0.5 & 2.13 & 1.09 \\
\hline & Chifeng & $\mathrm{C} 1$ & 4.13 & 282 & 3.26 & 0 & 2.08 & 0.49 \\
\hline & & $\mathrm{C} 2$ & 3.37 & 164 & 3.39 & 1.1 & 1.93 & 0.48 \\
\hline & & C3 & 2.04 & 107 & 3.71 & 2.1 & 1.86 & 1.11 \\
\hline
\end{tabular}

\footnotetext{
${ }^{1}$ Degrees Theurer.

${ }^{2}$ Measured at $20^{\circ} \mathrm{C}$.
} 
Table 2. Information inferred from $16 \mathrm{~S}$ rRNA and internal transcribed spacer analyses and diversity indices of koumiss samples from Inner Mongolia

\begin{tabular}{|c|c|c|c|c|c|c|c|c|}
\hline Target & Sample $^{1}$ & $\begin{array}{l}\text { No. of } \\
\text { reads }\end{array}$ & $\begin{array}{l}\text { No. of } \\
\text { OTU }^{2}\end{array}$ & $\begin{array}{l}\text { Chao1 } \\
\text { index }\end{array}$ & $\begin{array}{l}\text { Shannon } \\
\text { index }\end{array}$ & $\begin{array}{l}\text { Simpson } \\
\text { index }\end{array}$ & $\begin{array}{l}\text { Good's } \\
\text { coverage }\end{array}$ & $\begin{array}{l}\text { No. of observed } \\
\text { species }\end{array}$ \\
\hline \multirow[t]{8}{*}{ Bacterial analysis } & $\mathrm{X} 1$ & 22,916 & 241 & 380 & 3.78 & 0.87 & 0.9989 & 241 \\
\hline & $\mathrm{X} 2$ & 33,992 & 178 & 221 & 3.97 & 0.90 & 0.9996 & 178 \\
\hline & $\mathrm{X} 4$ & 26,578 & 265 & 330 & 3.88 & 0.87 & 0.9992 & 265 \\
\hline & H1 & 21,098 & 232 & 278 & 3.59 & 0.84 & 0.9992 & 232 \\
\hline & H2 & 32,188 & 185 & 254 & 2.45 & 0.59 & 0.9995 & 185 \\
\hline & C1 & 29,305 & 232 & 310 & 2.68 & 0.73 & 0.9993 & 232 \\
\hline & $\mathrm{C} 2$ & 29,512 & 251 & 276 & 2.80 & 0.76 & 0.9995 & 251 \\
\hline & C3 & 28,118 & 295 & 382 & 4.26 & 0.90 & 0.9990 & 295 \\
\hline \multirow[t]{6}{*}{ Fungal analysis } & $\mathrm{X} 1$ & 11,579 & 85 & 106 & 1.53 & 0.36 & 0.9998 & 85 \\
\hline & $\mathrm{X} 2$ & 14,747 & 102 & 130 & 2.25 & 0.59 & 0.9999 & 102 \\
\hline & X3 & 15,009 & 89 & 120 & 2.45 & 0.72 & 0.9998 & 89 \\
\hline & $\mathrm{C} 1$ & 10,121 & 90 & 128 & 3.47 & 0.84 & 0.9996 & 90 \\
\hline & $\mathrm{C} 2$ & 12,988 & 97 & 100 & 3.26 & 0.79 & 0.9999 & 97 \\
\hline & C3 & 14,119 & 92 & 115 & 2.21 & 0.69 & 0.9998 & 92 \\
\hline
\end{tabular}

${ }^{1}$ See Table 1 for abbreviation definitions.

${ }^{2}$ Operational taxonomic units.

was compared by the Chao1 index. The Chao1 index of bacteria $(315 \pm 61)$ was significantly higher than that of fungi $(108 \pm 20)$ in koumiss from Inner Mongolia $(P$ $<0.01$ ). The bacterial and fungal diversity of koumiss was compared by the Shannon and Simpson indices. The Shannon index of bacteria $(3.63 \pm 0.72)$ was significantly higher than that of fungi $(2.27 \pm 1.07 ; P<$ $0.01)$. The Simpson index of bacteria $(0.83 \pm 0.1)$ was significantly higher than that of fungi $(0.6 \pm 0.27 ; P<$ $0.05)$. Similarly, the observed species of bacteria (248 \pm 48) were significantly more numerous than that of fungi $(84 \pm 14 ; P<0.01)$. The above comparative alpha indicators illustrated that koumiss from Inner Mongolia had more bacterial phylotypes than fungus. In contrast, in tarag, a bacteria- and fungi-containing dairy product produced by traditional fermentation of cow milk in Inner Mongolia, the bacterial diversity was significantly lower than the diversity of fungi (Sun et al., 2014). We speculated that the difference in nutritional profile between mare and cow milks (e.g., high lactose content of mare milk) may arouse the predominance of bacteria or fungi in traditional fermented dairy products.

The samples were obtained from 11 banners in 3 administrative divisions of Inner Mongolia (Figure 1). The Kruskal-Wallis and Tukey HSD analyses were applied to evaluate the differences in the bacterial and fungal richness and diversity of koumiss from 3 areas (X: Xilin Gol; H: Hulun Buir; C: Chifeng). The results
$(P>0.05)$ showed that there was no difference between these 3 divisions (Figure 3). Due to the inheritance of artisanal technique of traditional production of koumiss among Mongols in these 3 divisions, the procedure of spontaneous fermentation of koumiss and the nutritional and microbiological profiles of mare milk from the Mongolian breed of horse were prone to be relatively consistent, which imparted different divisions with stable microbial richness and diversity in koumiss.

\section{Bacterial Community Profile of Koumiss}

Based on the 16S rRNA sequences, the bacterial phyla were identified as belonging to 6 phyla-namely, Firmicutes (relative abundance: 64.45\%), Proteobacteria (34.55\%), Actinobacteria (0.91\%), Bacteroidetes (0.07\%), Deinococcus-Thermus (0.01\%), and Cyanobacteria (0.01\%). Of the 6 bacteria phyla, Firmicutes dominated in 7 samples $(\mathrm{X} 1, \mathrm{X} 3, \mathrm{X} 4, \mathrm{H} 1, \mathrm{H} 4, \mathrm{C} 1$, and C3), corresponding to $79.08,74.34,91.34,94.71,82.15$, 61.48 , and $79.38 \%$ of the reads from 3 divisions of Inner Mongolia, respectively. In contrast, Proteobacteria dominated in the remaining 4 samples (X2, H2, H3, and $\mathrm{C} 2$ ), corresponding to $60.5,78.01,51.1$, and $54.87 \%$ of the reads, respectively. The above results were consistent with previous studies that demonstrated 4 major bacterial phyla (Firmicutes, Proteobacteria, Actinobacteria, and Bacteroidetes) in koumiss (Gesudu et al., 
2016), kefir (Dobson et al., 2011), and tarag (Sun et al., 2014), which are traditional fermented dairy products, and Firmicutes dominated in the bacterial community. In this study, Proteobacteria was the predominant bacterial phylum in 4 samples, which was different from the previous studies. These results implied that the transformation of predominant phylum could take place in different samples of artisanal koumiss from spontaneous fermentation.

As shown in Figure 4A, 126 bacterial genera were identified; the number of genera $>1 \%$ was 8 , including Lactobacillus (50.91\%), Acetobacter (17.99\%), Exiguobacterium (8.17\%), Lactococcus (7.62\%), Kleb- siella $(5.75 \%)$, Acinetobacter $(2.69 \%)$, Pseudomonas (1.75\%), and Enterobacter (1.67\%). Lactobacillus was the predominant bacterial genus. It is well known that Lactobacillus plays a significant role in fermented dairy products (Karami et al., 2017a; Wang et al., 2018). Of the 126 bacteria genera, Lactobacillus dominated in 7 samples (X1, X3, X4, H1, H4, C1, and C3), corresponding to $77.45,61.91,70.02,93.57,77.66,52.51$, and $58.67 \%$ of the reads from these 3 areas, respectively. The richness of Lactobacillus determined the predominant phylum of Firmicutes. In contrast, Acetobacter and Lactobacillus dominated in $\mathrm{H} 2$ and $\mathrm{H} 3$, corresponding to 63.06 and $19.2 \%$ for $\mathrm{H} 2$ and 27.1 and $26.18 \%$ for
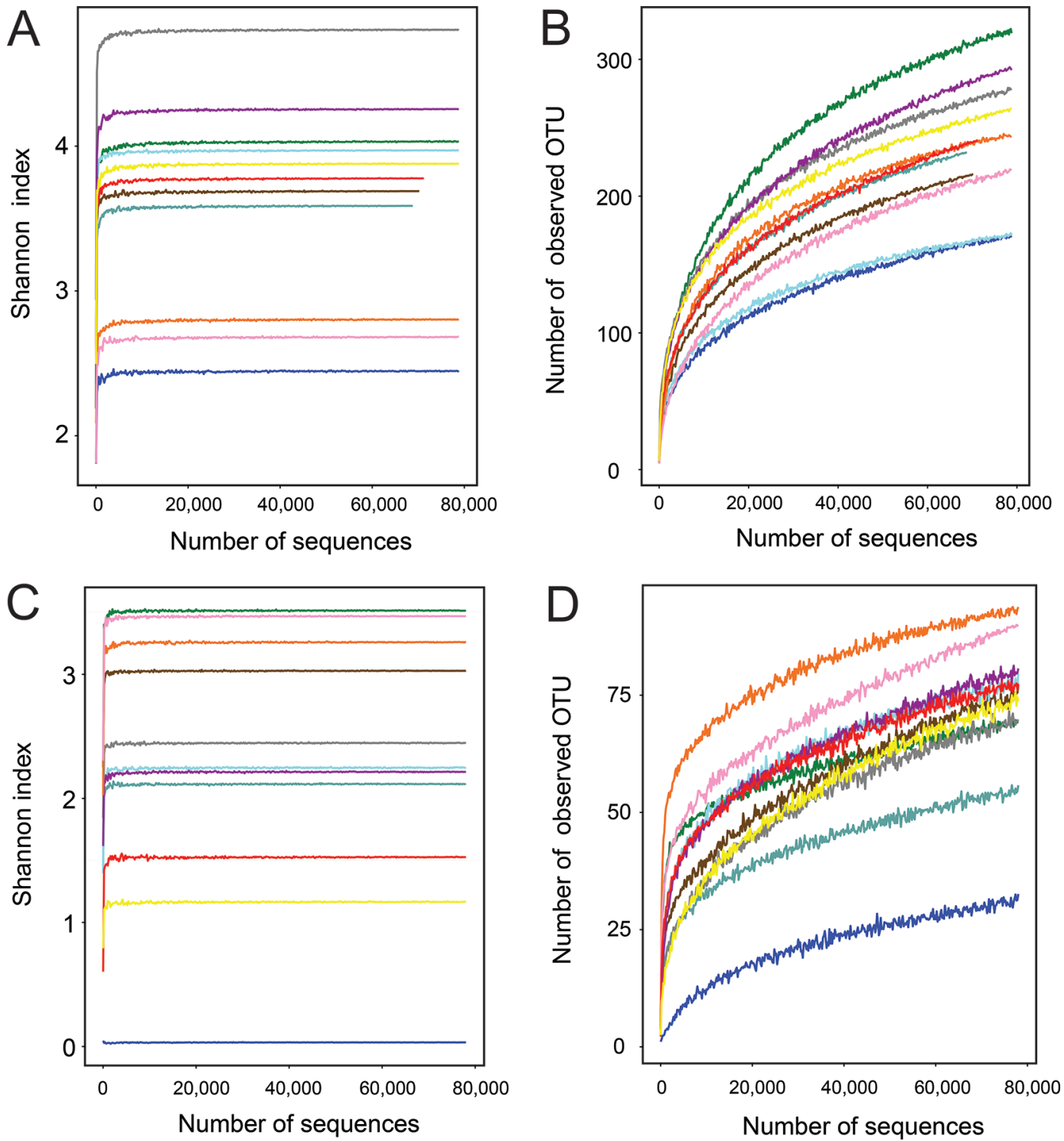

Figure 2. Shannon diversity curves (A and C) and rarefaction curves (B and D) for 16S rRNA (A and B) and internal transcribed spacer $(\mathrm{C}$ and $\mathrm{D})$ sequencing analyses. OTU = operational taxonomic units. 

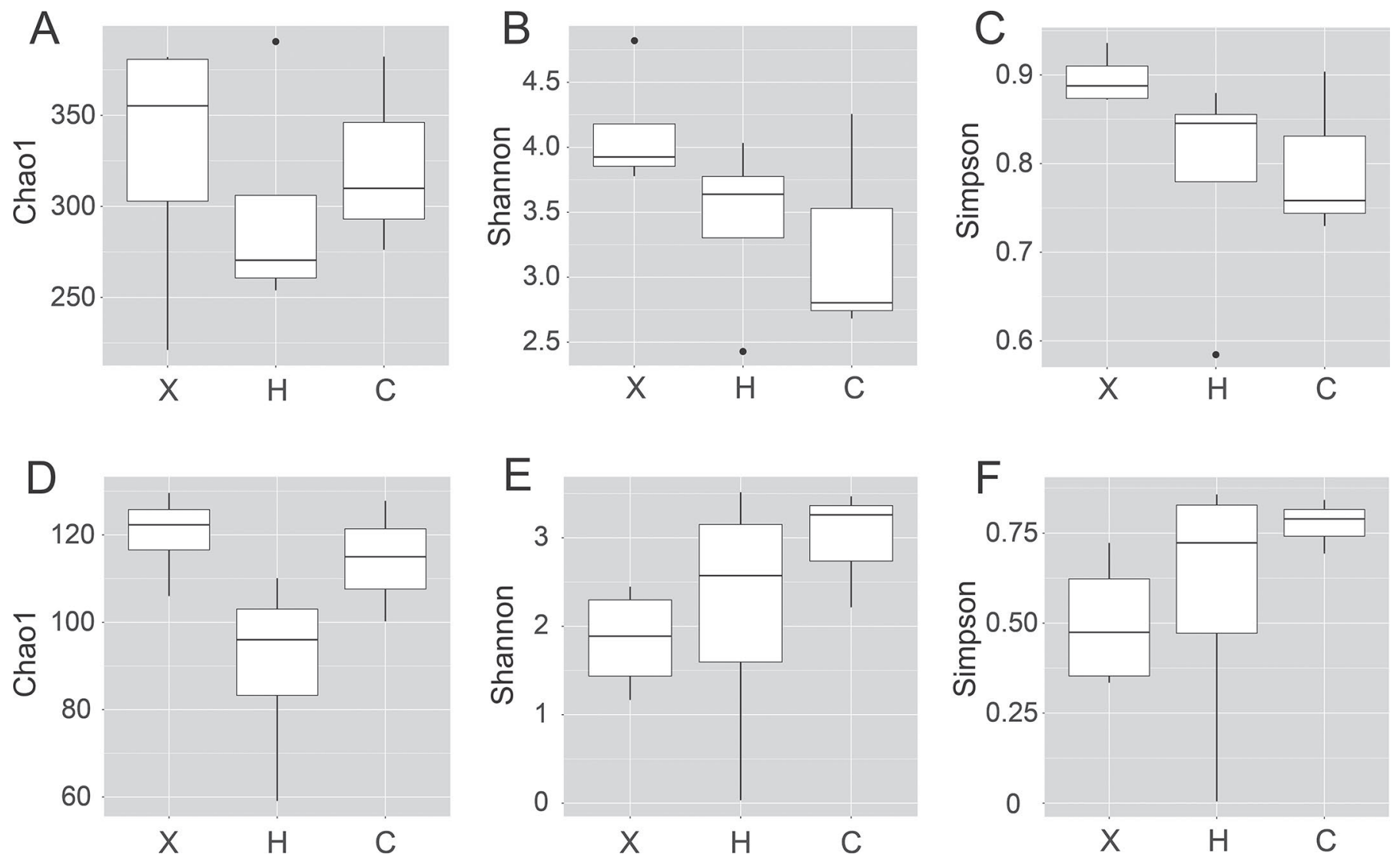

Figure 3. Box plots showing the comparisons of Chao1 (A and D), Shannon (B and E), and Simpson (C and F) indices from 3 administrative divisions (X: Xilin Gol; H: Hulun Buir; C: Chifeng) of Inner Mongolia for 16S rRNA (A, B, and C) and internal transcribed spacer (D, E, and F) sequencing analyses. Box plot is a graphic representation of a distribution of value by a rectangle, the ends of which mark the first and third quartiles, and the maximum and minimum values are marked by whiskers. The middle line marks the median and outliers are marked by a dot.

H3, respectively. Due to the ability of Acetobacter with alcohol dehydrogenase to oxidize ethanol to acetic acid (Tian et al., 2017), Acetobacter was able to transform alcohol produced by yeasts to acetic acid. Lactococcus and Klebsiella dominated in X2, corresponding to 21.9 and $27.41 \%$, respectively. Lactococcus is regarded as an important dairy microorganism (Casalta and Montel, 2008; Cavanagh et al., 2015). Although Klebsiella is a potential pathogen for humans and ruminants, Klebsiella species together with Lactobacillus could enhance the functional potential of fermented dairy products (Zadoks et al., 2011; Rosales-Bravo et al., 2017). Exiguobacterium and Acinetobacter dominated in C2, corresponding to 40.69 and $13.03 \%$, respectively. The bacterial genus Exiguobacterium possesses stress-responsive genes that help it colonize and thrive in diverse ecological niches (Kasana and Pandey, 2018) and can be found in Latin-style cheeses (Lusk et al., 2012). These results suggested that the bacterial genus varied in 11 koumiss samples from 3 divisions of Inner Mongolia. Due to spontaneous fermentation of koumiss in the individual's yurt, microbiological qualities of mare milk and traditional starter culture and sanitary conditions may influence bacterial community structure.

At the species level, 49 bacteria species were identified; the number of species $>1 \%$ was 8 , including $L c$. lactis (61.58\%), Lactobacillus buchneri (12.72\%), Enterococcus italicus (5.87\%), Lactobacillus homohiochii (5.12\%), Lactobacillus hilgardii (4.88\%), Lb. helveticus $(2.56 \%)$, Leuconostoc mesenteroides $(2.34 \%)$, and Streptococcus parauberis $(2.32 \%)$. Of the 49 bacterial species, Lc. lactis dominated in 4 samples (X2, X3, X4, and C3), corresponding to $76.68,88.84,95.12$, and $85.42 \%$ of the reads from 3 divisions of Inner Mongolia, respectively. Lactococcus lactis and Strep. parauberis dominated in $\mathrm{H} 3$, corresponding to 38.57 and $29.31 \%$, respectively. Lactobacillus helveticus dominated in 3 samples (X1, $\mathrm{H} 1$, and H4), corresponding to 57.18, 57.81, and $75.16 \%$, respectively; $L b$. buchneri dominated in $\mathrm{H} 2$ (53.46\%); and Lb. hilgardii dominated in C1 (54.08\%). Streptococcus thermophilus and Ent. italicus dominated in C2, corresponding to 20.68 and $41.65 \%$, respectively. 
The microbes that belonged to lactic acid bacteria were predominant in koumiss in this study and exhibited a multifaceted significant function in fermented dairy products in the previous studies, such as starter cultures (Vinderola et al., 2002; Takeda et al., 2011; Yu et al., 2011), antimicrobial activity (Ayeni et al., 2009; Arqués et al., 2015; Karami et al., 2017b), and probiotics and health promoting (Kamiya et al., 2016; Kimoto-Nira et al., 2017; Linares et al., 2017).

The varieties and relative abundance of bacteria genera and species were clearly different in different samples that were obtained from 11 banners in the 3
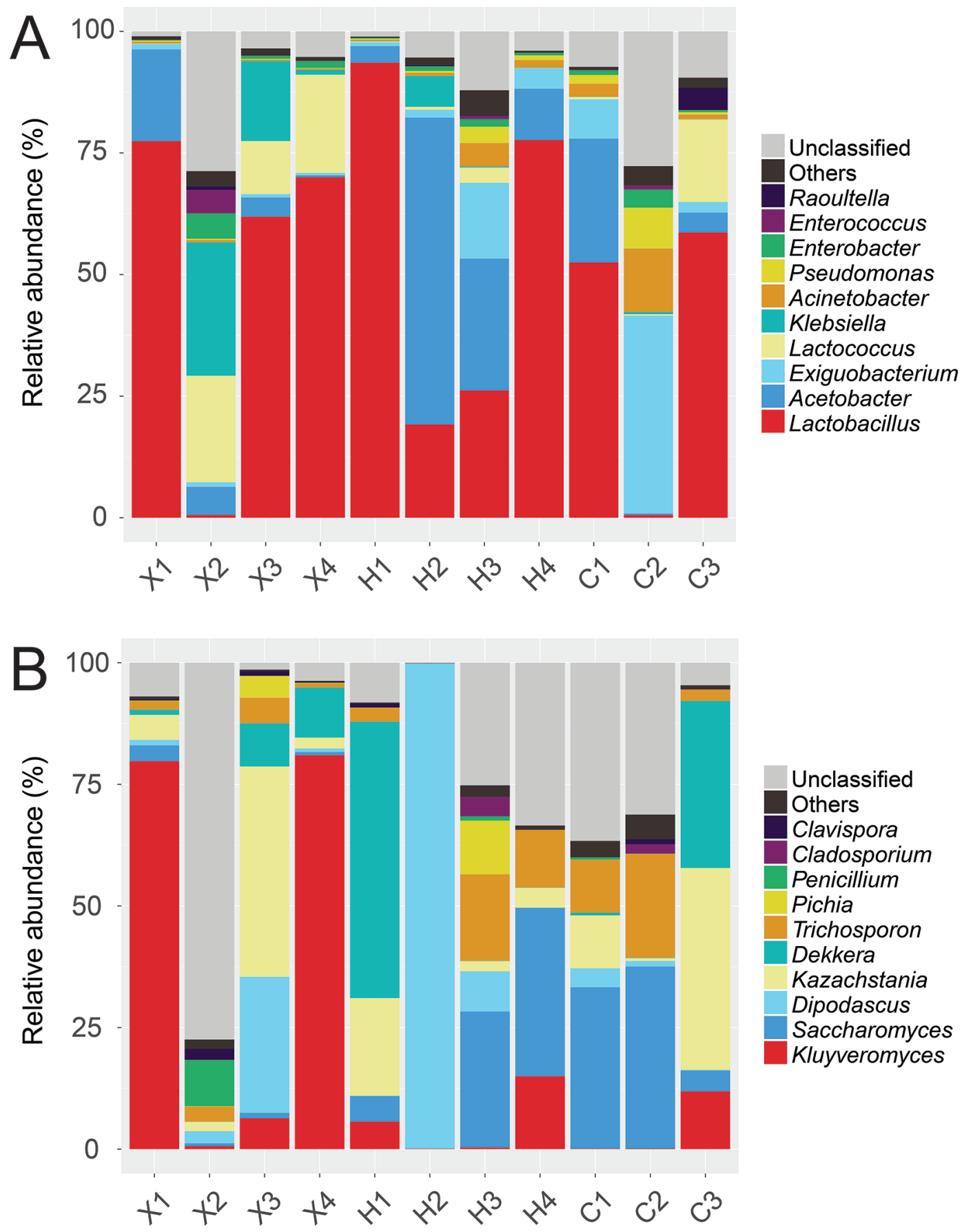

Figure 4. Relative abundance of bacterial (A) and fungal (B) sequences at the genus level in each koumiss sample from 3 administrative divisions (X: Xilin Gol, 4 samples; H: Hulun Buir, 4 samples; C: Chifeng, 3 samples) of Inner Mongolia. 
administrative divisions. The above result of diversity corresponded to the previous study, which focused on the variation in the bacterial diversity in koumiss from different geographic locations (Gesudu et al., 2016). In terms of the overall technique of traditional fermentation of koumiss in Inner Mongolia, we speculated (1) that microorganisms in homemade starters, raw mare milk, environment, and appliances for fermentation were sources of microbial flora in koumiss and (2) that the nutritional quality of mare milk and artisanal technological parameters for production (e.g., temperature, time of duration for fermentation, and intensity of beating and stirring) likely influenced the varieties of bacterial and fungal communities in end-product koumiss from different areas. The bacterial community structures of koumiss from 3 divisions were compared as inferred from the unweighted and weighted UniFrac $\mathrm{PCoA}$. As demonstrated in Figure 5A and B, the values of 11 samples from 3 divisions were largely separated in the both unweighted (accounting for 26.25 and $16.61 \%$ of the total variance by the 2 principal components, respectively) and weighted (accounting for 60.58 and $15.34 \%$ of the total variance by the 2 principal components, respectively) analyses. In addition, the Kruskal-Wallis and Tukey HSD analyses which were based on the unweighted and weighted UniFrac PCoA were applied to evaluate the differences in the bacterial community structures of koumiss from 3 areas (X: Xilin Gol; H: Hulun Buir; C: Chifeng). As shown in Figure $6 \mathrm{~A}$ and $\mathrm{B}$, the results $(P>0.05)$ showed that there was no difference in the bacterial community structure of koumiss from these 3 areas. When the sequence data of individual samples were pooled for the comparisons between each division, there was no difference in the bacterial diversity of koumiss from these 3 divisions. Due to neighboring and no distinct geographical barrier between these 3 divisions, the bacterial community structures of 11 koumiss samples were not significantly different.

\section{Fungal Community Profile of Koumiss}

Based on the ITS sequences, the fungal phyla were identified as belonging to 3 phyla - namely, Ascomycota (relative abundance: 91.64\%), Basidiomycota (8.35\%), and Mucoromycota (0.01\%). Ascomycota (91.64 \pm $8.22 \%$ ) dominated all koumiss samples from Inner Mongolia. In previous studies, the predominant phylum was identified as Ascomycota in traditional dairy products (Sun et al., 2014; Gao et al., 2017) and beverages containing alcohol (Jung et al., 2012; Li et al., 2013).

As shown in Figure 4A, 59 fungal genera were identified; the number of genera $>1 \%$ was 11 , including Kluyveromyces (20.11 $\pm 32.75 \%)$, Saccharomyces (19.47 $\pm 23.89 \%)$, Dipodascus $(14.59 \pm 29.51 \%)$, Kazachstania $(13.85 \pm 16.21 \%)$, Dekkera $(10.94 \pm 20.03 \%)$, Trichosporon $(10.63 \pm 10.6 \%)$, Penicillium $(4.03 \pm 12.65 \%)$, Pichia $(1.81 \pm 4.5 \%)$, Clavispora $(1.27 \pm 2.95 \%)$, Cladosporium $(0.78 \pm 1.73 \%)$, and Aspergillus (0.57 $\pm 0.66 \%$ ). Of the 59 fungal genera, Kluyveromyces dominated in 2 samples (X1 and X4), corresponding to 85.72 and $84.12 \%$ of the reads from these 3 areas, respectively. Saccharomyces and Trichosporon dominated in 4 samples ( $\mathrm{H} 3, \mathrm{H} 4, \mathrm{C} 1$, and $\mathrm{C} 2)$, corresponding to $61.1,69.8,69.57$, and $85.37 \%$, respectively. Kazachstania $(43.86 \%)$ and Dipodascus $(28.35 \%)$ dominated in X3, and Kazachstania (43.47\%) and Dekkera (36.03\%) dominated in C3. Dekkera (61.92\%) dominated in H1, and Dipodascus (99.79\%) dominated in H2. These predominant genera in 10 samples belong to yeasts, and Kluyveromyces, Saccharomyces, Dipodascus, Kazachstania, and Dekkera play important roles in the processes of proteolysis, lipolysis, flavor emergence, lactic acid utilization, alcohol production, and fermentation of dairy products (Narvhus and Gadaga, 2003; Landell et al., 2006; Sudun et al., 2013; Sun et al., 2014). Nonetheless, Penicillium (42.14\%), a genus of filamentous fungus, was the major genus in X2. Moreover, all 11 samples contained Penicillium, Cladosporium, and Aspergillus, which were regarded as commonly filamentous fungi leading to dairy spoilage such as off odor and flavor and dairy waste as well as economic losses (Garnier et al., 2017). The presence of spoilage fungi in koumiss indicated that the fermentation of mare milk required the sanitation of tools and the tight control of environmental cleaning and hygiene. Similar to the diversity of bacterial genera in koumiss, the varieties and abundance of fungal genera were different among these 11 koumiss. We speculated that the microbiological profiles of raw milk and traditional starter culture and environmental and tool hygiene were likely to affect the fungal community structure of traditional koumiss.

At the species level, 57 fungal species were identified; the number of genera $>1 \%$ was 8 , including Kluyveromyces marxianus (25.22\%), Kazachstania unispora (19.8\%), Dekkera anomala (17.66\%), Saccharomyces cerevisiae (17.55\%), Trichosporon asahii (10.25\%), Penicillium carneum (2.83\%), Pichia membranifaciens $(2.05 \%)$, and Clavispora lusitaniae (1.13\%). Similar to the analysis of bacterial community, the varieties and relative abundance of fungal species were clearly distinct in individual samples. The variation in fungal diversity was also discovered in spontaneously fermented cow milk from different geographic locations (Sun et al., 2014). The unweighted and weighted UniFrac PCoA were introduced to compare the fungal community structures of koumiss from 3 divisions, and the values of 11 samples from these 3 divisions were largely 

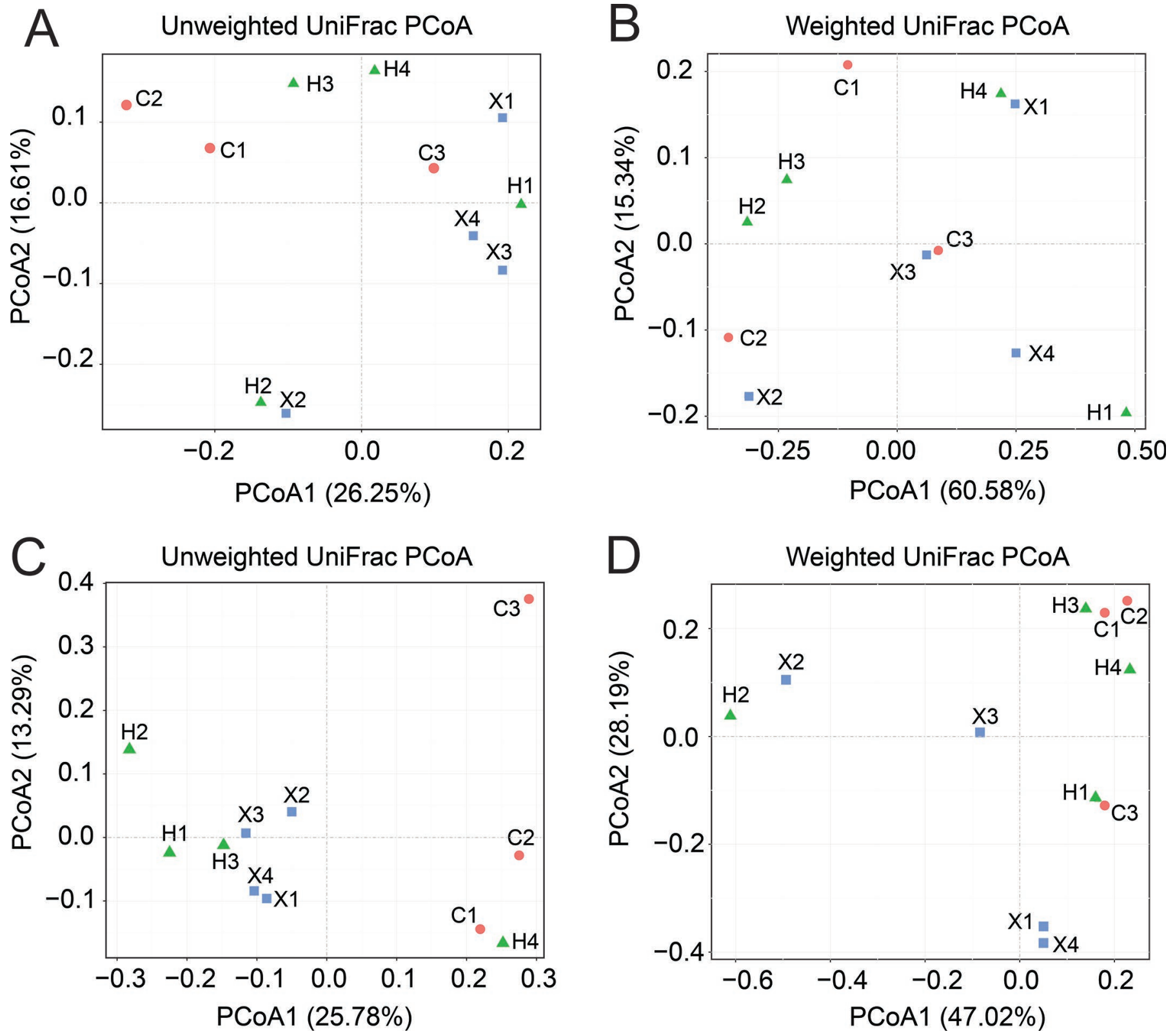

Figure 5. Unweighted (A and $\mathrm{C}$ ) and weighted (B and D) UniFrac principal coordinate analyses (PCoA) of the bacterial (A and B) and fungal (C and D) diversity in 11 koumiss samples from 3 administrative divisions of Inner Mongolia. The blue (square), green (triangle), and red (circle) symbols represent the samples from Xilin Gol (X; $\mathrm{n}=4)$, Hulun Buir $(\mathrm{H} ; \mathrm{n}=4)$, and Chifeng $(\mathrm{C} ; \mathrm{n}=3)$, respectively, in Inner Mongolia.

separated in the both unweighted (accounting for 25.78 and $13.29 \%$ of the total variance by the 2 principal components, respectively) and weighted (accounting for 47.02 and $28.19 \%$ of the total variance by the 2 principal components, respectively) analyses (Figure 5C and D). Moreover, we used the Kruskal-Wallis and Tukey HSD analyses, which were based on the unweighted and weighted UniFrac PCoA of the sequence data of the samples from different areas, to evaluate the differences in the fungal community structure of koumiss from 3 areas (X: Xilin Gol; H: Hulun Buir; C: Chifeng). The results $(P>0.05)$ demonstrated that there was no difference between the 3 neighboring divisions without a distinct geographical barrier.

\section{CONCLUSIONS}

Simultaneous investigation of bacterial and fungal community structures in traditional koumiss is a prerequisite for understanding the mechanisms of sponta- 

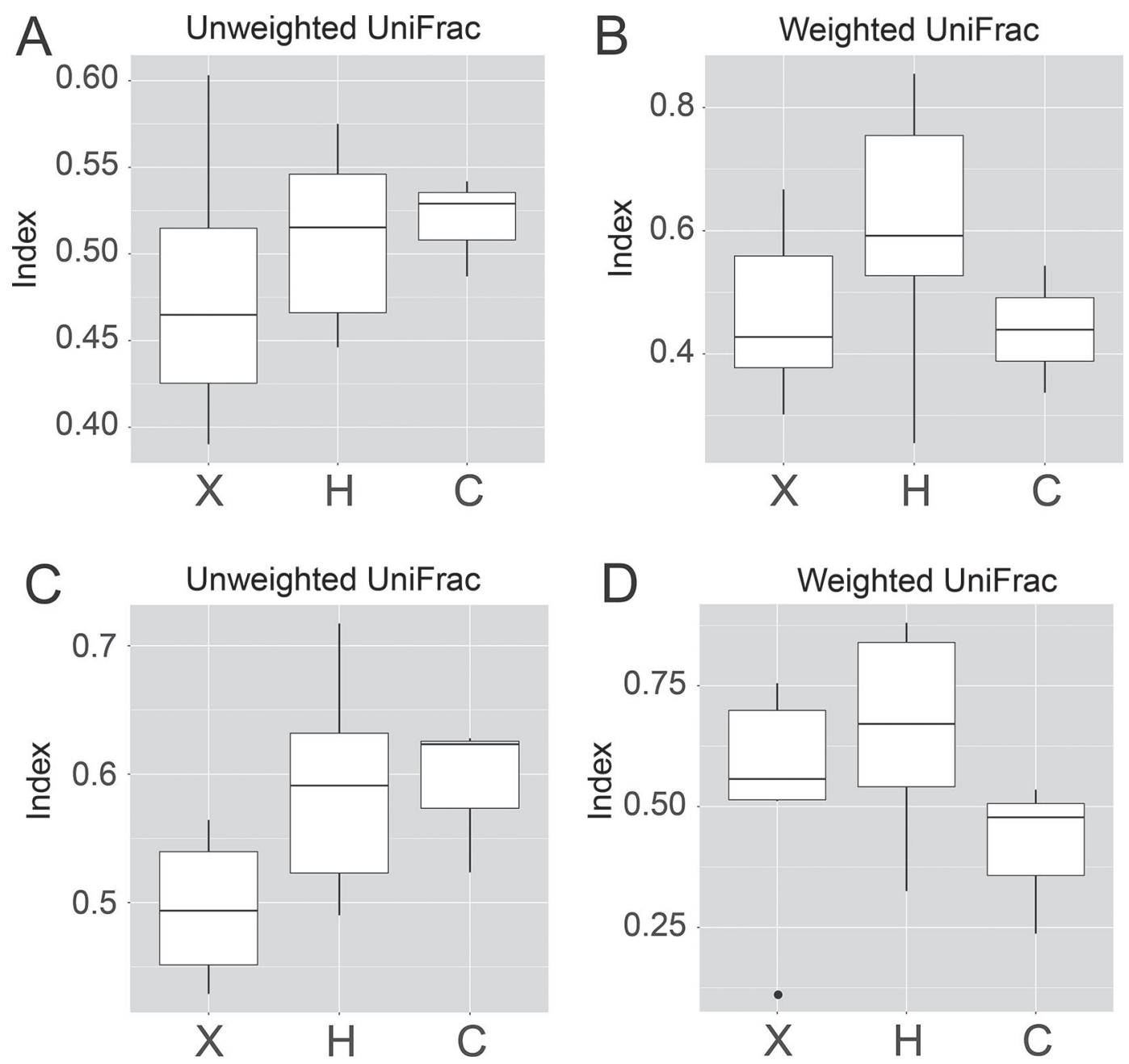

Figure 6. Box plots showing the comparisons of unweighted ( $\mathrm{A}$ and $\mathrm{C}$ ) and weighted (B and D) UniFrac principal coordinate analyses of the bacterial (A and B) and fungal (C and D) diversity from 3 areas (X: Xilin Gol; H: Hulun Buir; C: Chifeng) of Inner Mongolia. Box plot is a graphic representation of a distribution of value by a rectangle, the ends of which mark the first and third quartiles, and the maximum and minimum values are marked by whiskers. The middle line marks the median and outliers are marked by a dot.

neous fermentation of koumiss, which is fundamental for the development of commercial starter cultures for industrial and standardized production of koumiss in the future. In this study, the bacterial and fungal florae were investigated in artisanal koumiss from Inner Mongolia using high-throughput sequencing. First, nutritional analyses illustrated that the acidity and the alcohol content significantly increased with the decreasing of lactose content during traditional fermentation of koumiss. Then, our results demonstrated that 6 bacterial phyla represented by 126 genera and 49 species were discovered, and 3 fungal phyla represented by 59 genera and 57 species were verified in koumiss samples. Lactobacillus was the predominant genus of bacterium, and Kluyveromyces and Saccharomyces dominated the fungal genus overall in koumiss. Last, the varieties and relative abundance of bacterial and fungal genera and species were obviously distinct in individual samples, and there were no differences in the bacterial and fungal richness and diversity of koumiss from the 3 neighboring administrative areas in Inner Mongolia as inferred from the analysis of combined sequence data from all samples.

\section{ACKNOWLEDGMENTS}

We thank Miao-Miao Hao, Yun-Fang Wu, and Nanslama (Xilingol Vocational College, Xilinhot, China) for their help with the nutritional and microbiological experiments. We thank Yan Ju (Purdue University, West Lafayette, IN) for providing related references. Funding was provided by Xilin Gol Institute of Bioengineering 
Key Research Project (200601; Xilinhot, China) and Chinese National Key Research and Development Program (2017YFE0108800; Beijing, China).

\section{REFERENCES}

Arqués, J. L., E. Rodriguez, S. Langa, J. M. Landete, and M. Medina. 2015. Antimicrobial activity of lactic acid bacteria in dairy products and gut: Effect on pathogens. BioMed Res. Int. 2015:584183.

Ayeni, F. A., B. A. Adeniyi, S. T. Ogunbanwo, R. Tabasco, T. Paarup, C. Pelaez, and T. Requena. 2009. Inhibition of uropathogens by lactic acid bacteria isolated from dairy foods and cow's intestine in western Nigeria. Arch. Microbiol. 191:639-648.

Bokulich, N. A., S. Subramanian, J. J. Faith, D. Gevers, J. I. Gordon, R. Knight, D. A. Mills, and J. G. Caporaso. 2013. Quality-filtering vastly improves diversity estimates from Illumina amplicon sequencing. Nat. Methods 10:57-59.

Caporaso, J. G., J. Kuczynski, J. Stombaugh, K. Bittinger, F. D. Bushman, E. K. Costello, N. Fierer, A. G. Pena, J. K. Goodrich, J. I. Gordon, G. A. Huttley, S. T. Kelley, D. Knights, J. E. Koenig, R. E. Ley, C. A. Lozupone, D. McDonald, B. D. Muegge, M. Pirrung, J. Reeder, J. R. Sevinsky, P. J. Turnbaugh, W. A. Walters, J. Widmann, T. Yatsunenko, J. Zaneveld, and R. Knight. 2010. QIIME allows analysis of high-throughput community sequencing data. Nat. Methods 7:335-336.

Casalta, E., and M. C. Montel. 2008. Safety assessment of dairy microorganisms: The Lactococcus genus. Int. J. Food Microbiol. 126:271-273.

Cavanagh, D., G. F. Fitzgerald, and O. McAuliffe. 2015. From field to fermentation: The origins of Lactococcus lactis and its domestication to the dairy environment. Food Microbiol. 47:45-61.

China National Food Safety Standard. 2010. GB 5413.5-2010. Determination of lactose and sucrose in foods for infants and young children. Ministry of Health of the People's Republic of China, Beijing, China.

China National Food Safety Standard. 2016a. GB 5009.239-2016. Determination of acidity in foods. Ministry of Health of the People's Republic of China, Beijing, China.

China National Food Safety Standard. 2016b. GB 5009.225-2016. Determination of alcohol in liquors. Ministry of Health of the People's Republic of China, Beijing, China.

Danova, S., K. K. Petrov, P. Pavlov, and P. Petrova. 2005. Isolation and characterization of Lactobacillus strains involved in koumiss fermentation. Int. J. Dairy Technol. 58:100-105.

Dobson, A., O. O'Sullivan, P. D. Cotter, P. Ross, and C. Hill. 2011. High-throughput sequence-based analysis of the bacterial composition of kefir and an associated kefir grain. FEMS Microbiol. Lett. 320:56-62.

Edgar, R. C. 2013. UPARSE: Highly accurate OTU sequences from microbial amplicon reads. Nat. Methods 10:996-998.

Gao, M. L., H. M. Hou, X. X. Teng, Y. L. Zhu, H. S. Hao, and G. L. Zhang. 2017. Microbial diversity in raw milk and traditional fermented dairy products (Hurood cheese and Jueke) from Inner Mongolia, China. Genet. Mol. Res. 16:1-13.

Garnier, L., F. Valence, and J. Mounier. 2017. Diversity and control of spoilage fungi in dairy products: An update. Microorganisms $5: 1-33$.

Gesudu, Q., Y. Zheng, X. Xi, Q. C. Hou, H. Xu, W. Huang, H. Zhang, B. Menghe, and W. Liu. 2016. Investigating bacterial population structure and dynamics in traditional koumiss from Inner Mongolia using single molecule real-time sequencing. J. Dairy Sci. 99:7852-7863.

Hao, Y., L. Zhao, H. Zhang, Z. Zhai, Y. Huang, X. Liu, and L. Zhang. 2010. Identification of the bacterial biodiversity in koumiss by denaturing gradient gel electrophoresis and species-specific polymerase chain reaction. J. Dairy Sci. 93:1926-1933.

Jung, M. J., Y. D. Nam, S. W. Roh, and J. W. Bae. 2012. Unexpected convergence of fungal and bacterial communities during fermentation of traditional Korean alcoholic beverages inoculated with various natural starters. Food Microbiol. 30:112-123.
Kamiya, T., Y. Watanabe, S. Makino, H. Kano, and N. M. Tsuji. 2016. Improvement of intestinal immune cell function by lactic acid bacteria for dairy products. Microorganisms 5:1-10.

Karami, S., M. Roayaei, H. Hamzavi, M. Bahmani, H. HassanzadAzar, M. Leila, and M. Rafieian-Kopaei. 2017a. Isolation and identification of probiotic Lactobacillus from local dairy and evaluating their antagonistic effect on pathogens. Int. J. Pharm. Investig. $7: 137-141$.

Karami, S., M. Roayaei, E. Zahedi, M. Bahmani, L. Mahmoodnia, H. Hamzavi, and M. Rafieian-Kopaei. 2017b. Antifungal effects of Lactobacillus species isolated from local dairy products. Int. J. Pharm. Investig. 7:77-81.

Kasana, R. C., and C. B. Pandey. 2018. Exiguobacterium: An overview of a versatile genus with potential in industry and agriculture. Crit. Rev. Biotechnol. 38:141-156.

Kimoto-Nira, H., N. Moriya, S. Hayakawa, K. Kuramasu, H. Ohmori, S. Yamasaki, and M. Ogawa. 2017. Effects of rare sugar D-allulose on acid production and probiotic activities of dairy lactic acid bacteria. J. Dairy Sci. 100:5936-5944.

Köljalg, U., K. H. Larsson, K. Abarenkov, R. H. Nilsson, I. J. Alexander, U. Eberhardt, S. Erland, K. Hoiland, R. Kjoller, E. Larsson, T. Pennanen, R. Sen, A. F. Taylor, L. Tedersoo, T. Vralstad, and B. M. Ursing. 2005. UNITE: A database providing web-based methods for the molecular identification of ectomycorrhizal fungi. New Phytol. 166:1063-1068.

Landell, M. F., C. Hartfelder, and P. Valente. 2006. Identification and enzymatic profile of yeasts isolated from artisanal cheese in Southern Brazil. Acta Sci. Vet. 31:49-55.

Li, X. R., E. B. Ma, L. Z. Yan, H. Meng, X. W. Du, and Z. X. Quan. 2013. Bacterial and fungal diversity in the starter production process of Fen liquor, a traditional Chinese liquor. J. Microbiol. 51:430-438.

Linares, D. M., C. Gomez, E. Renes, J. M. Fresno, M. E. Tornadijo, R. P. Ross, and C. Stanton. 2017. Lactic acid bacteria and bifidobacteria with potential to design natural biofunctional healthpromoting dairy foods. Front. Microbiol. 8:846.

Lusk, T. S., A. R. Ottesen, J. R. White, M. W. Allard, E. W. Brown, and J. A. Kase. 2012. Characterization of microflora in Latin-style cheeses by next-generation sequencing technology. BMC Microbiol. 12:254.

Magoč, T., and S. L. Salzberg. 2011. FLASH: Fast length adjustment of short reads to improve genome assemblies. Bioinformatics 27:2957-2963

Mu, Z., X. Yang, and H. Yuan. 2012. Detection and identification of wild yeast in koumiss. Food Microbiol. 31:301-308.

Narvhus, J. A., and T. H. Gadaga. 2003. The role of interaction between yeasts and lactic acid bacteria in African fermented milks: A review. Int. J. Food Microbiol. 86:51-60.

Pan, D. D., X. Q. Zeng, and Y. T. Yan. 2011. Characterisation of Lactobacillus fermentum SM-7 isolated from koumiss, a potential probiotic bacterium with cholesterol-lowering effects. J. Sci. Food Agric. 91:512-518.

Pruesse, E., C. Quast, K. Knittel, B. M. Fuchs, W. Ludwig, J. Peplies, and F. O. Glockner. 2007. SILVA: A comprehensive online resource for quality checked and aligned ribosomal RNA sequence data compatible with ARB. Nucleic Acids Res. 35:7188-7196.

Rong, J., H. Zheng, M. Liu, X. Hu, T. Wang, X. Zhang, F. Jin, and L. Wang. 2015. Probiotic and anti-inflammatory attributes of an isolate Lactobacillus helveticus NS8 from Mongolian fermented koumiss. BMC Microbiol. 15:196.

Rosales-Bravo, H., H. C. Morales-Torres, J. Vazquez-Martinez, J. Molina-Torres, V. Olalde-Portugal, and L. P. Partida-Martinez. 2017. Novel consortium of Klebsiella variicola and Lactobacillus species enhances the functional potential of fermented dairy products by increasing the availability of branched-chain amino acids and the amount of distinctive volatiles. J. Appl. Microbiol. 123:1237-1250.

Sudun, Wulijideligen, K. Arakawa, M. Miyamoto, and T. Miyamoto. 2013. Interaction between lactic acid bacteria and yeasts in airag, an alcoholic fermented milk. Anim. Sci. J. 84:66-74.

Sun, Z., W. Liu, Q. Bao, J. Zhang, Q. Hou, L. Kwok, T. Sun, and H. Zhang. 2014. Investigation of bacterial and fungal diversity in 
tarag using high-throughput sequencing. J. Dairy Sci. 97:60856096.

Takeda, S., K. Yamasaki, M. Takeshita, Y. Kikuchi, C. Tsend-Ayush, B. Dashnyam, A. M. Ahhmed, S. Kawahara, and M. Muguruma. 2011. The investigation of probiotic potential of lactic acid bacteria isolated from traditional Mongolian dairy products. Anim. Sci. J. 82:571-579

Tian, L., S. J. Perot, S. Hon, J. Zhou, X. Liang, J. T. Bouvier, A. M. Guss, D. G. Olson, and L. R. Lynd. 2017. Enhanced ethanol formation by Clostridium thermocellum via pyruvate decarboxylase. Microb. Cell Fact. 16:171.

Vinderola, C. G., P. Mocchiutti, and J. A. Reinheimer. 2002. Interactions among lactic acid starter and probiotic bacteria used for fermented dairy products. J. Dairy Sci. 85:721-729.

Wang, J., T. Wu, X. Fang, W. Min, and Z. Yang. 2018. Characterization and immunomodulatory activity of an exopolysaccharide produced by Lactobacillus plantarum JLK0142 isolated from fermented dairy tofu. Int. J. Biol. Macromol. 115:985-993.

Wang, Q., G. M. Garrity, J. M. Tiedje, and J. R. Cole. 2007. Naive Bayesian classifier for rapid assignment of rRNA sequences into the new bacterial taxonomy. Appl. Environ. Microbiol. 73:5261-5267.
Ya, T., Q. Zhang, F. Chu, J. Merritt, M. Bilige, T. Sun, R. Du, and H. Zhang. 2008. Immunological evaluation of Lactobacillus casei Zhang: A newly isolated strain from koumiss in Inner Mongolia, China. BMC Immunol. 9:68.

Yang, X., Y. Wang, Y. Zhou, X. Gao, B. Li, and G. Huo. 2014. Complete genome sequence of Lactococcus lactis ssp. lactis KLDS4. 0325, a bacterium newly isolated from koumiss in Xinjiang, China. Wei Sheng Wu Xue Bao 54:1406-1418.

Yi, L., Y. Dang, J. Wu, L. Zhang, X. Liu, B. Liu, Y. Zhou, and X. Lu. 2016. Purification and characterization of a novel bacteriocin produced by Lactobacillus crustorum MN047 isolated from koumiss from Xinjiang, China. J. Dairy Sci. 99:7002-7015.

Yu, J., W. H. Wang, B. L. Menghe, M. T. Jiri, H. M. Wang, W. J. Liu, Q. H. Bao, Q. Lu, J. C. Zhang, F. Wang, H. Y. Xu, T. S. Sun and H. P. Zhang. 2011. Diversity of lactic acid bacteria associated with traditional fermented dairy products in Mongolia. J. Dairy Sci. 94:3229-3241.

Zadoks, R. N., H. M. Griffiths, M. A. Munoz, C. Ahlstrom, G. J. Bennett, E. Thomas, and Y. H. Schukken. 2011. Sources of Klebsiella and Raoultella species on dairy farms: Be careful where you walk. J. Dairy Sci. 94:1045-1051. 